\title{
Theoretical quantification of shape distortion in fuzzy Hough transform ${ }^{\text {is }}$
}

\author{
Jayanta Basak ${ }^{\mathrm{a}, *}$, Sankar K. Pal ${ }^{\mathrm{b}}$ \\ ${ }^{a}$ IBM India Research Laboratory, Block-I, Indian Institute of Technology, Hauz Khas, New Delhi 110016, India

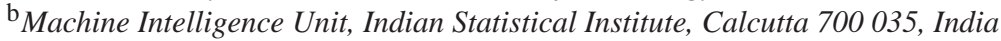

Received 27 February 2004; received in revised form 28 December 2004; accepted 23 February 2005

Available online 14 March 2005

\begin{abstract}
We present a generalization of classical Hough transform in fuzzy set theoretic framework (called fuzzy Hough transform or FHT) in order to handle the impreciseness/ill-definedness in shape description. In addition to identifying the shapes, the methodology can quantify the amount of distortion present in each shape by suitably characterizing the parametric space. We extended FHT to take care of gray level images (gray FHT) in order to handle the gray level variation along with shape distortion. The gray FHT gives rise to a scheme for image segmentation based on the a priori knowledge about the shapes.

(c) 2005 Elsevier B.V. All rights reserved.
\end{abstract}

Keywords: Hough transform; Fuzzy Hough transform; Shape distortion; Entropy

\section{Introduction}

Hough transform was originally invented by Hough [13] and later refined [10] to detect analytically defined shapes like straight lines, circles, ellipses, etc. from binary images. Later it has been extended to generalized Hough transform $[1,2]$ in order to detect arbitrary shapes characterized by a set of shape features. Different problems of image analysis and computer vision [10,2,27], including edge/line linking,

\footnotetext{
This work has been done while the first author was in the machine intelligence unit, Indian Statistical Institute, Calcutta, India.

* Corresponding author.

E-mail address: bjayanta@in.ibm.com (J. Basak).
} 
boundary detection, motion detection, object recognition and shape analysis exploit the concept of Hough transform and generalized Hough transform. One of the basic advantages of Hough transform is that it works even for the broken edge and line segments, i.e., in the presence of small gaps (loss of connectivity).

Hough transform (and generalized Hough transform) maps shapes like lines, circles, ellipses etc. (and arbitrary shapes defined a priori as a collection of features in the case of generalized Hough transform) from the image space to a parameter space in which the shapes are represented in terms of their parameter values. Each point on a shape in the image space is transformed into a curve in the parameter space, and all the points belonging to a particular shape are transformed to a family of curves passing through a common point (in the ideal condition). The point of intersection of these family of curves represents the set of parameters defining the shape. In general, a cluster is formed in the parameter space corresponding to a shape in the image space. The edges/lines or curves of general shapes can therefore be detected by separating out these clusters (peaks) in the parameter space.

In real-life images, the shapes are often distorted from their true parametric forms due to the presence of noise, digitization error and shape variations. Even if the input image is perfect, it is often very difficult to describe the shapes in terms of the perfect model shapes. For example, the real-life objects can be described as approximately circular or approximately elliptical which often do not lend themselves to precise shape properties. Again the gray values in the object region also vary widely due to the effect of external illumination. In other words, shapes become ill-defined in terms of grayness and spatial ambiguities. Therefore, it may be difficult to find out a single peak or a compact cluster in the parameter space after applying Hough transform. In other words, a single shape in an image may get broken into more than one local peak in the parameter space. This will, in turn, result in the decrease in the amplitude of the peaks in the parameter space; thereby causing difficulty in their detection. Even if these multiple peaks can be identified, they will essentially represent multiple shapes leading to a misinterpretation.

In order to take care of these difficulties of classical Hough transform, several variations have already been introduced depending on the type of applications. A state-of-the-art survey [14,21,19] describes the different implementation techniques and variations of classical Hough transform. The other algorithmic variations of Hough transform include randomized Hough transform [28], probabilistic Hough transform [16-18,8] and fuzzy Hough transform [9,12,22,11,25,26,23]. In randomized Hough transform, certain object pixels are randomly chosen in the image space and based on certain criteria (for example, the local gradient information), parameter values are obtained for the shapes. Although the randomized Hough transform is computationally very fast, it is prone to noise because the local gradient information is affected by noise or distorted shapes. In the probabilistic Hough transform, in general, a subset of the object pixels in an image is randomly selected and the parameter values are computed based on these selected points. Different criteria are set for choosing the subset randomly. It effectively reduces the computation time. However, the probabilistic Hough transform also has the same disadvantages as that of classical one for identifying shapes in the presence of noise or under distortion.

The fuzzy Hough transform (FHT) is based on the realization that a shape does not lend itself to a precise definition because of grayness and spatial ambiguities, and therefore it can be considered as a fuzzy set where each pixel has a degree of possessing certain imprecise shape property represented by the set. Han, Koczy and Poston developed a fuzzy Hough transform algorithm [12] for finding out shapes by approximately fitting the data points to some given parametric shapes. For this purpose, around each point on the perfect shape defined by the parametric form, a small region was defined, and each pixel in this region, i.e., each pixel in the vicinity of the perfect shape contributes to the accumulator space. Thus the accumulator values exhibit a smooth transition from the higher to lower values so that the peaks can 
be identified unambiguously even for certain distorted image shapes. A faster computation of the same was also proposed in [12] by convolving the accumulator space with Gaussian window. Philip et al. [22] described a fuzzy Hough transform technique for extracting features based on a similar concept as in [12], where accumulator values were computed from a thick shaded zone around the distorted shape.

In both these algorithms [12,22], the distorted input image shapes were first fuzzified, (i.e., a thick shaded zone is considered) and then the corresponding accumulator values were computed. However, the extent to which the shapes need to be fuzzified, i.e., the amount of thickness of the shaded zone needs to be considered, was not specified in either of these algorithms. In identifying the shapes by fuzzy Hough transform, one needs to quantify the amount of vagueness present in the shapes (in terms of the extent of the shaded zone) as it is essential in the subsequent higher order decision making tasks e.g., object recognition [5], generation of visual description [7]. Moreover, it is often necessary to apply Hough transform directly on gray level images. In the classical Hough transform, usually a segmented version of the gray level image is considered, or the contribution of each pixel to the accumulator space is made proportional to the gray value of the pixel. It is difficult to take care of the distorted shapes and the presence of noise in the classical framework. The situation becomes even worse when noise amplitude is comparable to the average gray level of the shapes.

These problems have been taken care of in our generalized fuzzy set theoretic framework of Hough transform. Note that, in [12,22], no generalization of the FHT algorithm for the gray images was provided, and also no guideline for selecting the optimal thickness of the shaded zone was specified. In defining FHT, first, the classical Hough transform has been interpreted in a set theoretic framework and then a generalization in terms of fuzzy set theory is made. The model shapes (e.g., straight lines, circles, etc.) are fuzzified and represented as fuzzy shaded zones (Fig. 1) instead of having single pixel thick lines/curve segments. Each pixel in the shaded zone has a membership value which reflects the degree to which a pixel possesses the corresponding shape property. The pixels lying on the core line of the shaded zone possess maximum membership value and those in the periphery have zero membership values. Therefore, each shape is given a notion of approximate shape. For example, instead of finding a circle in an image, as performed by the HT, the FHT essentially peaks out an approximate circle in the image. The degree of approximation, in turn, depends on the amount of fuzzification introduced on the model shapes. A measure is defined to indicate the suitability of fuzzification and based on this measure, an optimum amount of fuzzification is derived. The vagueness or ambiguity in the shape description due to the fuzzification is also quantified.

We then extended the concept of the FHT to gray images by fuzzifying the gray shapes to an optimum extent. In the domain of gray images, variations of HT have been proposed [24,20] where the gray values of the pixels are handled either by digital halftoning the image [24] or by adding an extra dimension to the accumulator space [20]. In the case of the FHT for gray images (called Gray FHT), an optimum gray level threshold is found for each shape along with the thickness of the shaded zone. Since the gray level threshold depends on the average gray value of the specific shapes, this generalization leads to a gray level image segmentation scheme guided by the knowledge about the parametric form of the shapes.

The rest of the article is organized as follows. Section 2 describes the classical Hough transform in brief. In Section 3, a set theoretic interpretation of the Hough transform is presented which is then generalized to the fuzzy set theoretic framework (i.e., fuzzy Hough transform or FHT), based on the fuzzification of model shapes. In Section 4, we quantify the amount of fuzzification of the model shapes necessary for proper identification of ill-defined shapes. Section 5 extends the concept of FHT to gray images. The experimental results are illustrated in Section 6. Finally, Section 7 concludes the article. 


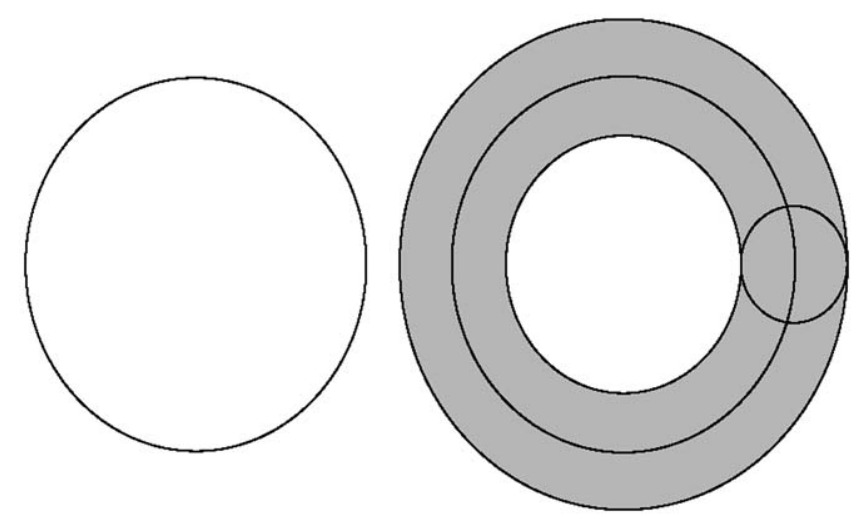

Fig. 1. A perfect circle is fuzzified as shown by the shaded zone. The core line of the shaded zone corresponds to the model shape. The small circle inside the shaded zone represents a fuzzified region corresponding to the central point on the core line.

\section{Classical Hough transform}

In the case of Hough transform, pixels lying on a curve/line segment in an image are transformed from the image space to the parameter space representing the analytical (mathematical) form of the curve/line segment. Let a curve/line segment be analytically defined as

$$
f(x, y, \Theta)=0
$$

where $(x, y)$ are the coordinates of a pixel on the curve/line segment with respect to some standard reference frame.

$$
\Theta=\left(\theta_{1}, \theta_{2}, \ldots, \theta_{n}\right)
$$

is the set of parameters describing the shape of the curve/line segment. For example, a straight line can be described as

$$
r=x \cos \alpha+y \sin \alpha,
$$

where $(r, \alpha)$ are the parameters describing the straight line, $r$ is the perpendicular distance of the line segment from the origin, and $\alpha$, the angle subtended by the normal with respect to the $x$-axis. Similarly, a circle can be described as

$$
r^{2}=(x-a)^{2}+(y-b)^{2}
$$

where $(r, a, b)$ are the parameters describing the circle, $r$ is the radius and $(a, b)$ are the coordinates of the center.

In the classical Hough transform, given the parametric form of a curve in an image, all possible sets of parameter values are computed for each object pixel lying on the curve/line segment. The parameter space is quantized and an accumulator array $(A)$ is defined. The detection of curve/line segment using classical Hough transform can be algorithmically described as follows [2].

Step 1: Quantize the parameter space in the limits

$$
\theta_{1_{\min }} \leqslant \theta_{1} \leqslant \theta_{1_{\max }}, \theta_{2_{\min }} \leqslant \theta_{2} \leqslant \theta_{2_{\max }}, \ldots, \theta_{n_{\min }} \leqslant \theta_{n} \leqslant \theta_{n_{\max }} .
$$


Form an array $A\left(\theta_{1}, \theta_{2}, \ldots, \theta_{n}\right)$ whose elements are initially set to zero. This array is often termed as accumulator array.

Step 2: For each object pixel $(x, y)$ in the image, update the accumulator array as

$$
A\left(\theta_{1}, \theta_{2}, \ldots, \theta_{n}\right)=A\left(\theta_{1}, \theta_{2}, \ldots, \theta_{n}\right)+c(x, y)
$$

where $\theta_{1}, \theta_{2}, \ldots, \theta_{n}$ are the parameter values of the curve/line segment which pass through the point $(x, y)$ in the image. $c(x, y)$ is the contribution of the pixel $(x, y)$ to the accumulator space. For example, in a two-tone image, $c(x, y)$ is unity. Similarly, in a gray image, $c(x, y)$ can be taken as the normalized gray value of the pixel, and in a gradient image $c(x, y)$ is the gradient magnitude at $(x, y)$.

Step 3: Identify the local peaks in $A(\Theta)$ such that for every peak $\Theta_{i}, A\left(\Theta_{i}\right) \geqslant A_{0}, A_{0}$ being a certain threshold.

Note that, the classical Hough transform only converts an image into an accumulator or parameter space. Each point in the image space gives rise to a curve in the parametric space. In other words, a curve/line segment in the image is transformed into a family of curves in the parameter space. Ideally, these family of curves will pass through a single point in the parameter space which represents the parameter values describing the curve/line segment in the image space. Therefore, the accumulator value will be maximum in the quantization slot of parameter space which contains this point through which the family of curves are passing. In order to detect a curve/line segment in the image, the local maxima in the accumulator space, after thresholding, need to be detected.

In real-life, due to the effect of noise, digitization error and shape variations, the family of curves in the parameter space may not pass through a single point, rather a small region may be identified through which the family of curves will pass. Therefore, it depends on the size of quantization slots whether a single slot can be identified in the quantized parameter space or not. If the sizes of the quantization slots are large then a single slot may be identified but there will be difficulty in specifying the exact parameter values describing the curve/line segment in the image space. On the other hand, for small slot size, there can be more than one local maxima in the close vicinity arising from a single curve/line segment in the image space. In other words, a single peak (which is ideally expected) in the accumulator space may be broken into a number of smaller peaks.

Therefore using the classical Hough transform with precise shape definitions, it is often difficult to specify the exact parameter values and the location of the curve/line segments in an image. Also a single curve/line segment may be detected as more than one curve/line segment due to the preciseness in the model shape description. In the following section, we describe a generalized version of Hough transform in fuzzy set theoretic framework. This can deal with different types of vagueness/uncertainties arising from the effect of noise, digitization error, and shape and gray level variations.

\section{Fuzzy set theoretic framework for Hough transform (FHT)}

Let us first provide a set theoretic interpretation of Hough transform where each model shape is viewed as a crisp set of points. This interpretation is then extended in the light of fuzzy set theory in order to handle the imprecise/ill-defined shapes both in two-tone and gray-tone images. 


\subsection{Set theoretic interpretation of Hough transform}

Let us consider a binary image space defined as

$$
\mathbf{X}=\left\{(x, y) \mid x_{\min } \leqslant x \leqslant x_{\max }, y_{\min } \leqslant y \leqslant y_{\max }\right\} .
$$

Let a model shape $M$ in $\mathbf{X}$ with parameter set $\Theta$ be specified by a set of points $S$ such that

$$
S=\{(x, y) \mid f(x, y, \Theta)=0\},
$$

where $f(\cdot)$ is the parametric form of the model shape (see Eq. (1)).

Let the object region $(I \subset \mathbf{X})$ be defined as

$$
I=\{(x, y) \mid \text { gray value of }(x, y) \text { is unity }\} .
$$

Then Hough transform can be viewed as a mapping

$$
T: I \stackrel{f}{\longrightarrow} A,
$$

where $A$ is a set given as

$$
A=\left\{\left(\Theta_{k}, v_{k}\right)\left|v_{k}=\right| S_{k} \mid, k=0, \ldots, m\right\} .
$$

$\Theta_{k}$ is the $k$ th quantization slot in the accumulator space, $m$ is the maximum number of such slots. $\left|S_{k}\right|$ is the cardinality of the set $S_{k}$ which is defined as

$$
S_{k}=\left\{(x, y) \mid(x, y) \in I \bigwedge f\left(x, y, \Theta_{k}\right)=0\right\} .
$$

Let us now define the fuzzy Hough transform separately for both two-tone and gray-tone images. In two-tone images, the FHT takes care of the random variation in position of the pixels. In gray images, the variation in gray levels is taken care of, in addition.

\subsection{FHT in a two-tone image}

Let $S$ denote the set of points on a single pixel thick model shape M (e.g., ideal straight line, circle, ellipse, etc.) having a definite shape property defined by parameters $\Theta=\left(\theta_{1}, \ldots, \theta_{n}\right)$. Let $\mathcal{M}$ represent the fuzzified version of $M$, i.e., fuzzy straight line, fuzzy circle etc. For any point $(x, y) \in \mathbf{I}$ in the image, the membership of $(x, y)$ to the fuzzy set $\mathcal{M}$ is defined as

$$
\mu_{\mathcal{M}}^{\Theta, \Delta r}(x, y)=\max _{\forall\left(x_{0}, y_{0}\right) \in S}\left\{h\left((x, y) ;\left(x_{0}, y_{0}\right), \Delta r\right)\right\} .
$$

$h(\cdot)$ is a two-dimensional extension (Fig. 2) of Zadeh's $\pi$-function defined as

$$
h\left((x, y) ;\left(x_{0}, y_{0}\right), \Delta r\right)= \begin{cases}1-2\left(\frac{d}{\Delta r}\right)^{2} & \text { if } 0 \leqslant d<\frac{\Delta r}{2}, \\ 2\left(1-\frac{d}{\Delta r}\right)^{2} & \text { if } \frac{\Delta r}{2} \leqslant d<\Delta r \\ 0 & \text { if } d \geqslant \Delta r\end{cases}
$$




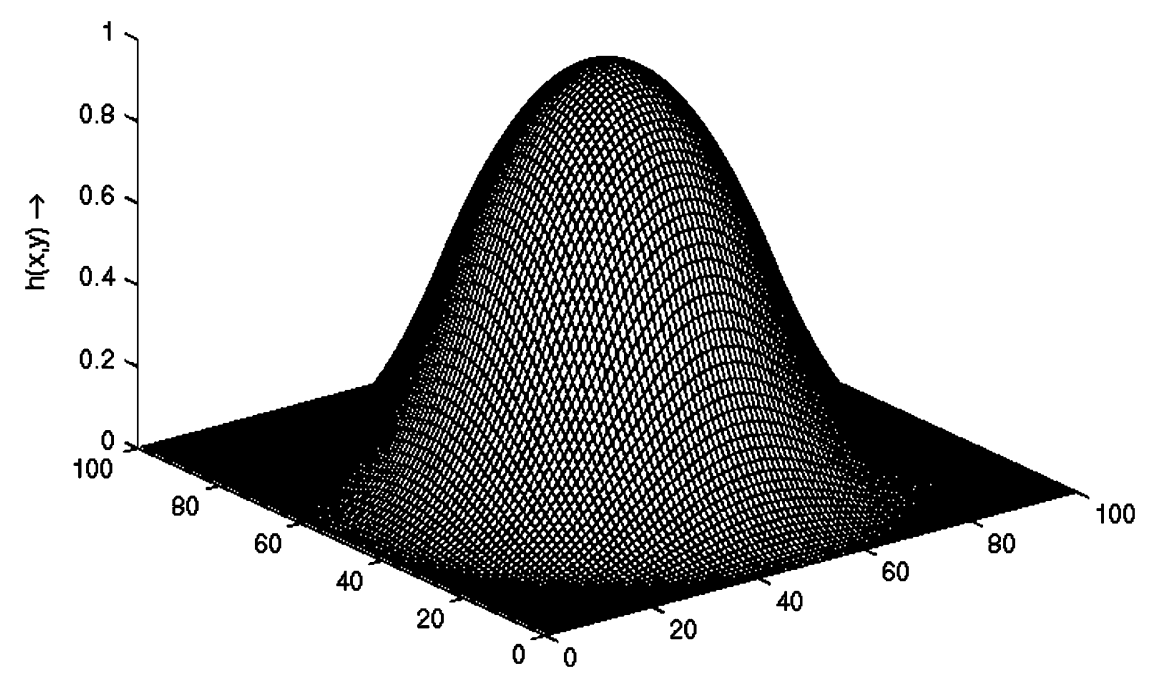

Fig. 2. A two-dimensional pi-function with the central point $\left(x_{0}, y_{0}\right)=(50,50)$ and bandwidth $\Delta r=50$.

where $d$ is the Euclidean distance of $(x, y)$ from $\left(x_{0}, y_{0}\right)$ such that

$$
d=\sqrt{\left(x-x_{0}\right)^{2}+\left(y-y_{0}\right)^{2}} .
$$

$\left(x_{0}, y_{0}\right)$ is the central point, i.e., the point where $h(\cdot)=1$. For all $\left(x_{0}, y_{0}\right)$ on the model shape $M, h(\cdot)=1$. $\Delta r$ is the bandwidth of the $\pi$-function such that $h(\cdot)=0.5$ when $d=\Delta r / 2$. In other words, $\Delta r$ reflects the maximum amount of deviation that a pixel can have from the ideal model shape.

The accumulator values are computed by

$$
A\left(\theta_{1}, \theta_{2}, \ldots, \theta_{n}, \Delta r\right)=\sum_{(x, y) \in \mathbf{X}} \mu_{\mathcal{M}}^{\Theta, \Delta r}(x, y) .
$$

The accumulator values indicate the contributions from all pixels which are in the vicinity (at a maximum distance of $\Delta r$ ) of the model shape $M$. The contribution of a pixel to the accumulator slot corresponding to $\mathcal{M}$ decreases as the distance of the pixel from $M$ increases. The set of pixels which lie within a distance $\Delta r$ from $M$ constitutes the supporting points of the fuzzy set $\mathcal{M}$. The corresponding region where these supporting points lie is defined as

$$
R=\left\{(x, y) \mid(x, y) \in \mathbf{I}, \quad \mu_{\mathcal{M}}^{\Theta, \Delta r}(x, y)>0\right\} .
$$

The spread of the accumulator values depends on the spread of the region $R$ which is described in Section 4.

Let us now explain the aforesaid generalized framework for the FHT with two different examples on circles and straight lines.

\subsubsection{FHT for a circle}

Let $C$ denote the set of points on a circle with center $(c 1, c 2)$ and radius $r$. Let $\mathcal{C}$ be the corresponding fuzzy set. In that case, for any point $(x, y) \in \mathbf{X}$ in the image, its membership on $\mathcal{C}$ is defined as

$$
\mu_{\mathcal{C}}^{c 1, c 2, r, \Delta r}(x, y)=g(d(x, y), \Delta r)
$$


where $d(x, y)$ is given as

$$
d(x, y)=\sqrt{\left((x-c 1)^{2}+(y-c 2)^{2}\right)}-r .
$$

$g(\cdot)$ is a $\pi$-function defined as

$$
g(d, \Delta r)= \begin{cases}0 & \text { if }|d|>\Delta r \\ 2\left(1-\frac{|d|}{\Delta r}\right)^{2} & \text { if } \frac{\Delta r}{2}<|d| \leqslant \Delta r \\ 1-2\left(\frac{d}{\Delta r}\right)^{2} & \text { if }|d| \leqslant \frac{\Delta r}{2}\end{cases}
$$

The accumulator values are computed by

$$
A(c 1, c 2, r, \Delta r)=\sum_{(x, y) \in \mathbf{X}} \mu_{\mathcal{C}}^{c 1, c 2, r, \Delta r}(x, y)
$$

for all parameter values.

\subsubsection{FHT for a straight line}

Let $L$ denote the set of points on a straight line with parameters $(r, \alpha)$. Let $\mathcal{L}$ be the corresponding fuzzy set. In that case, for any point $(x, y) \in \mathbf{X}$ in the image, its membership on $\mathcal{L}$ is defined as

$$
\mu_{\mathcal{L}}^{r, \alpha, \Delta r}(x, y)=g(d(x, y), \Delta r),
$$

$g(\cdot)$ being a $\pi$-function as defined in (17). The distance $d(x, y)$ of a point from the line segment is given as

$$
d(x, y)=x \cos \alpha+y \sin \alpha-r .
$$

The accumulator values are computed by

$$
A(r, \alpha, \Delta r)=\sum_{(x, y) \in \mathbf{X}} \mu_{\mathcal{L}}^{r, \alpha, \Delta r}(x, y)
$$

for all parameter values.

\subsection{FHT in a gray-tone image}

In a gray image, the object and background regions are not defined separately. The classical Hough transform can be directly applied to a gray level image where the contribution of each pixel in the accumulator space is equal to its normalized gray value. However, if the analytical shape is not perfect then the accumulator values will not smoothly decrease around the peak. Again the pixels can have variation in their gray values. The gray values of the pixels can be higher or lower than the average gray value of the actual shape. If the classical Hough transform is directly applied to a gray level image then the pixels with gray values higher than the average will contribute more to the accumulator space. In other 
words, the contribution of the noisy pixels will be more compared to the pixels which are representative of the true shape. As a result, the peak in the accumulator space may get shifted from its actual position, and the true shape may not be detected at all if the average gray value of that shape is comparable to the noise amplitude.

In applying Hough transform on gray level images, the average gray value of the shapes (objects) needs to be detected which may provide an useful information in the higher order visual information processing tasks. In addition, the thickness of the objects need also to be detected. In this section, a fuzzy set theoretic framework of HT is defined for gray images in order to handle the aforesaid problems.

Let $S$ denote the set of points on a single pixel thick model M (e.g., straight line, circle, ellipse etc.) having a definite shape property with parameters $\Theta=\left(\theta_{1}, \ldots, \theta_{n}\right)$ and a uniform gray value $l_{0}$. For any image point $(x, y) \in \mathbf{X}$ in the image, the membership of $(x, y)$ to the fuzzy shape $\mathcal{M}$ is defined as

$$
\mu_{\mathcal{M}}^{\Theta, l_{0}}(x, y)=\max _{\forall\left(x_{0}, y_{0}\right) \in S}\left\{h\left((x, y) ;\left(x_{0}, y_{0}\right), \Delta r\right) \cdot h_{1}\left(l(x, y) ; l_{0}, \Delta l\right)\right\},
$$

where $l(x, y)$ is the gray value of the pixel $(x, y), \Delta r$ is the maximum deviation of the pixel $(x, y)$ from the precise model shape $M$, and $\Delta l$ is the maximum deviation of the gray value of a pixel from $l_{0}$ (the gray value of the model shape). The function $h(\cdot)$ has the same form as in (11). $h_{1}(\cdot)$ is also a pi-type function with a single variable $l$.

The accumulator values are computed in exactly the same way as in the case of two-tone images, however, here the accumulator also keeps track of the gray values, i.e.,

$$
A\left(\Theta, l_{0}\right)=\sum_{(x, y) \in \mathbf{X}} \mu_{\mathcal{M}}^{\Theta, l_{0}}(x, y) .
$$

In gray images, the FHT for circles can be computed with expressions analogous to Eq. (15), where membership values of pixels over gray circles are given as

$$
\mu(x, y)_{\mathcal{C}}^{c 1, c 2, r, l_{0}}=g(d(x, y), \Delta r) \cdot h_{1}\left(l(x, y), l_{0}, \Delta l\right),
$$

where $l(x, y)$ is the gray value of the pixel $(x, y)$.

Similarly, for straight line segments,

$$
\mu_{\mathcal{L}}^{r, \alpha, l_{0}}(x, y)=g(d(x, y), \Delta r) \cdot h_{1}\left(l(x, y), l_{0}, \Delta l\right) .
$$

\section{Selection of the bandwidth}

As seen from Eqs. (13) and (23), the accumulator values are dependent on the nature of the membership function $h(\cdot)$. As the bandwidth $(\Delta r)$ of the membership function decreases, the sharpness of the peaks in accumulator space increases, thereby causing less difficulty in their localization. However, under noisy condition (in the presence of distortion), the accumulator space may exhibit a large number of spurious undesirable peaks corresponding to a single candidate shape in the image space, thereby increasing the difficulty in identifying each true shape. On the other hand, as $\Delta r$ increases, the accumulator profile tends to be smooth. In other words, the chance of correctly localizing the true peaks decreases. Figs. 3(b)-(f) illustrate the profile of accumulator values (the nature of quantized parameter space) for increase in $\Delta r$ corresponding to Fig. 3(a). 

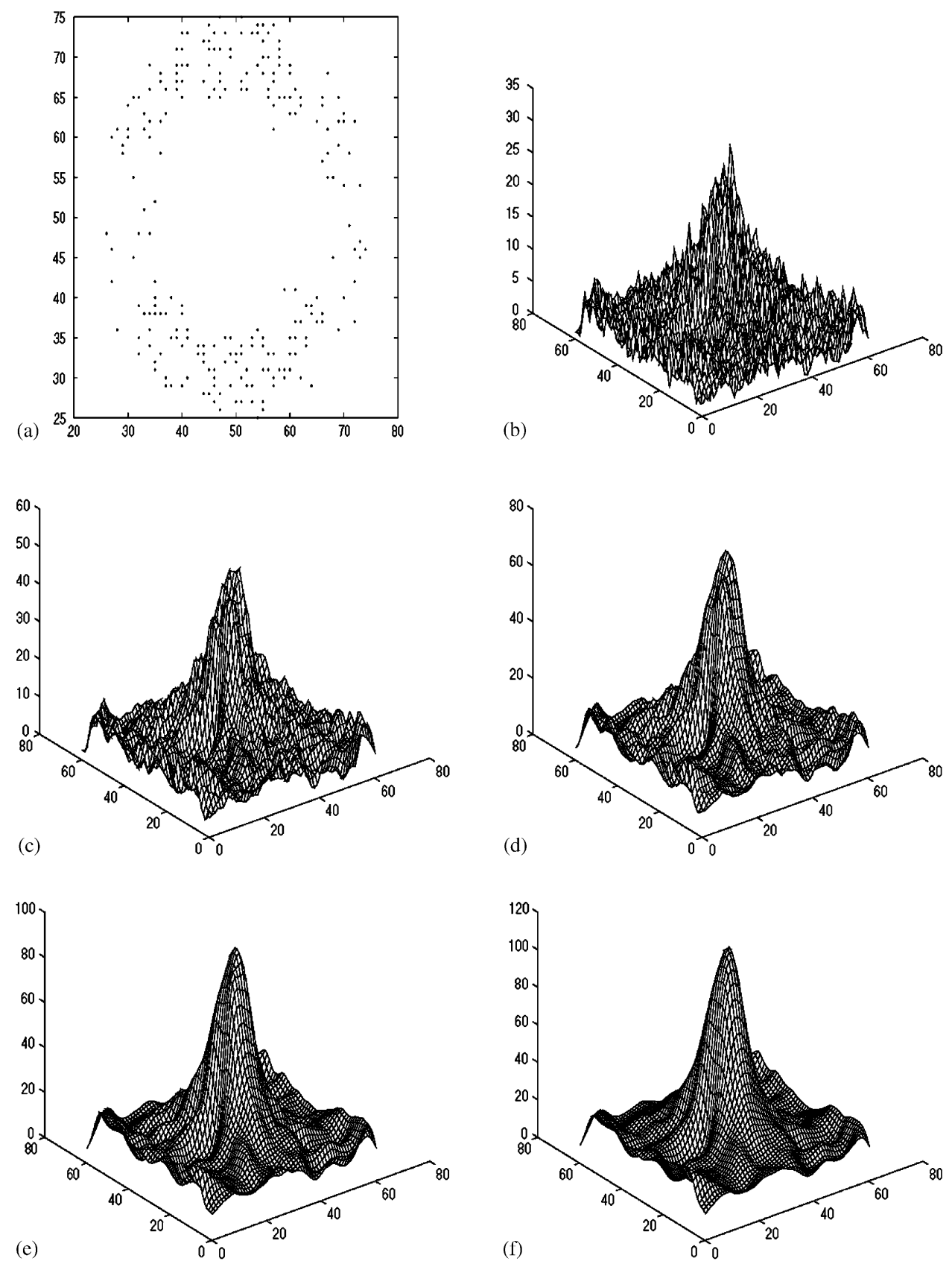

Fig. 3. Variation of the accumulator profile with the bandwidth. (a) The original image of a distorted/noisy circle with noise amplitude equal to 5, (b) the accumulator profile for $\Delta r=1$, (c) the accumulator profile with $\Delta r=2$, (d) the accumulator profile with $\Delta r=4$, (e) the accumulator profile with $\Delta r=5$, (f) the accumulator profile with $\Delta r=6$. 
Again, the bandwidth $\Delta r$ provides a measure of the amount of distortion of a shape in the image. This can further provide a useful measure in visual information representation and higher level visual information processing. It therefore, appears that the selection of bandwidth is crucial in shape identification. Such a selection of $\Delta r$ will also enable one to quantify the amount of distortion present in the shapes; thereby providing useful information in the higher level visual processes.

\subsection{For two-tone images}

As mentioned before, for a sharp peak, the accumulator profile will be highly compact around the peak. On the other hand, in the presence of flat accumulator profile or in the presence of multiple peaks in a single window, the compactness of the accumulator values will be low. In other words, if the bandwidth $(\Delta r)$ is not properly selected then the information content around the peak will be less and vice-versa. The information content can be quantified by various measures namely, linear index of fuzziness, quadratic index of fuzziness, Shannon's entropy and various others which are given as:

$$
\begin{aligned}
& L(\Theta, \Delta r)=\sum_{(x, y) \in \mathbf{X}} \min \left\{\mu_{\mathcal{M}}^{\Theta, \Delta r}(x, y), 1-\mu_{\mathcal{M}}^{\Theta, \Delta r}(x, y)\right\}, \\
& Q(\Theta, \Delta r)=\sum_{(x, y) \in \mathbf{X}} \mu_{\mathcal{M}}^{\Theta, \Delta r}(x, y)\left(1-\mu_{\mathcal{M}}^{\Theta, \Delta r}(x, y)\right)
\end{aligned}
$$

and

$$
H(\Theta, \Delta r)=-\sum_{(x, y) \in \mathbf{X}} \mu_{\mathcal{M}}^{\Theta, \Delta r}(x, y) \ln \mu_{\mathcal{M}}^{\Theta, \Delta r}(x, y) .
$$

Here $L, Q$, and $H$ are expressed as functions of $\Theta$ and $\Delta r$ because the index of fuzziness depends on both the bandwidth and the localization, i.e., where the model shape has been identified. Fig. 4 shows the variation of $L, Q$, and $H$ with respect to $\Delta r$ for a noisy image with noise amplitude equal to 4.0. Note that, here $\Theta$ is specified beforehand and the indices of fuzziness are measured with respect to the bandwidths only. It is observed that the information content is maximum for a certain value of $\Delta r$ which can be considered as the optimum $\Delta r$. It is also found experimentally that the optimum value of $\Delta r$ follows a linear relationship with the amount of distortion i.e., the noise amplitude (Fig. 5). The relationship between the optimum value of $\Delta r$ and the noise amplitude depends on the type of membership function and the noise pattern. Let us now provide a mathematical analysis of the relationship between the optimum value of $\Delta r$ and the amount of shape distortion.

\subsection{Theoretical analysis}

Since the linear index of fuzziness is not a smooth function, let us restrict the analysis to quadratic and Shannon entropy functions. Note that, the theoretical analysis can also be performed on other measures. For sake of simplicity, we restrict the analysis to quadratic and Shannon entropy functions.

Case I: The quadratic index of fuzziness is given as

$$
Q(\Theta, \Delta r)=\sum_{(x, y) \in \mathbf{X}} \mu_{\mathcal{M}}^{\Theta, \Delta r}(x, y)\left(1-\mu_{\mathcal{M}}^{\Theta, \Delta r}(x, y)\right) .
$$



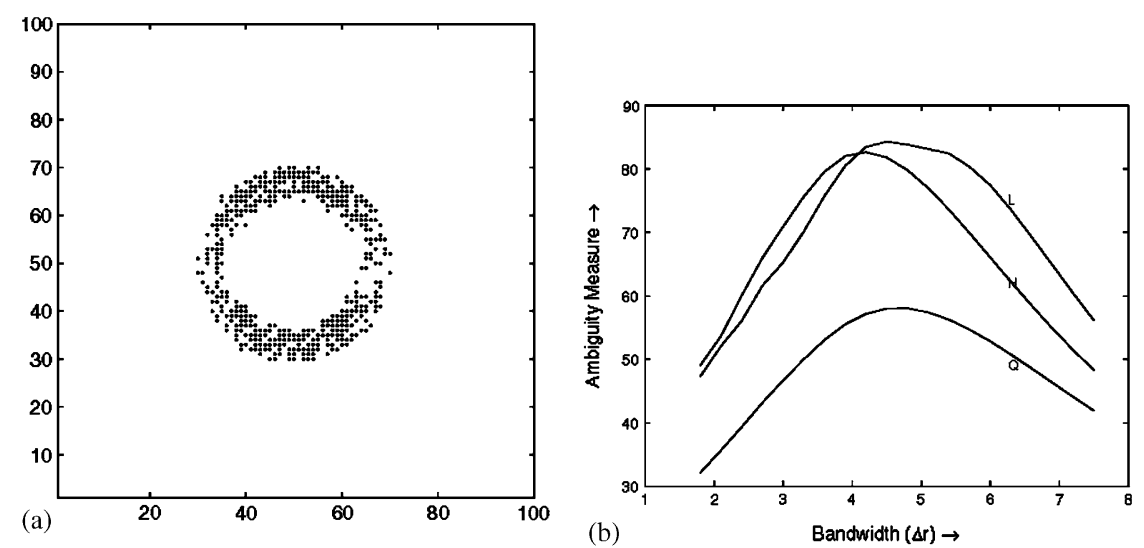

Fig. 4. (a) A noisy circle. (b) The variation of $L, H$, and $Q$ with the selected bandwidth $(\Delta r)$.

For a given parameter set $\Theta, Q$ is maximum when

$$
\frac{\partial Q}{\partial \Delta r}=0
$$

i.e.,

$$
\sum_{(x, y) \in \mathbf{X}}\left(1-2 \mu_{\mathcal{M}}^{\Theta, \Delta r}(x, y)\right) \frac{\partial \mu_{\mathcal{M}}^{\Theta, \Delta r}(x, y)}{\Delta r}=0 .
$$

Let us now assume that the membership values are modeled by a triangular function instead of a $\pi$-function such that

$$
\begin{aligned}
\mu_{\mathcal{M}}^{\Theta, \Delta r}(x, y) & =1-\frac{\delta_{\mathbf{x}}}{\Delta r} & & \text { for } \delta_{\mathbf{x}} \leqslant \Delta r, \\
& =0 & & \text { otherwise }
\end{aligned}
$$

where $\delta_{\mathbf{x}}$ is the minimum distance of the point $\mathbf{x}=(x, y)$ from the single pixel thick model shape $M$ characterized by the parameter set $\Theta$, i.e.,

$$
\delta_{\mathbf{x}}=\min _{\forall\left(x_{0}, y_{0}\right) \in S}\left\{\sqrt{\left(x-x_{0}\right)^{2}+\left(y-y_{0}\right)^{2}}\right\},
$$

where $S$ is the set of points in the image representing the model shape $M$. Therefore, from Eqs. (30)-(32),

$$
\Delta r_{\mathrm{opt}}=\frac{2 \sum_{(x, y) \in \Omega} \delta_{\mathbf{x}}^{2}}{\sum_{(x, y) \in \Omega} \delta_{\mathbf{x}}},
$$

where $\Delta r_{\text {opt }}$ is the optimum value of $\Delta r$ obtained from the maxima of $Q$, and $\Omega$ is a set such that $\delta_{\mathbf{x}}<\Delta r$, and $\Delta r_{\mathrm{opt}}$ is the optimum value of $\Delta r$. In other words, $\Omega$ is the set of supporting points such that

$$
\Omega=\left\{(x, y) \mid \mu_{\mathcal{M}}^{\Theta, \Delta r}(x, y)>0\right\} .
$$



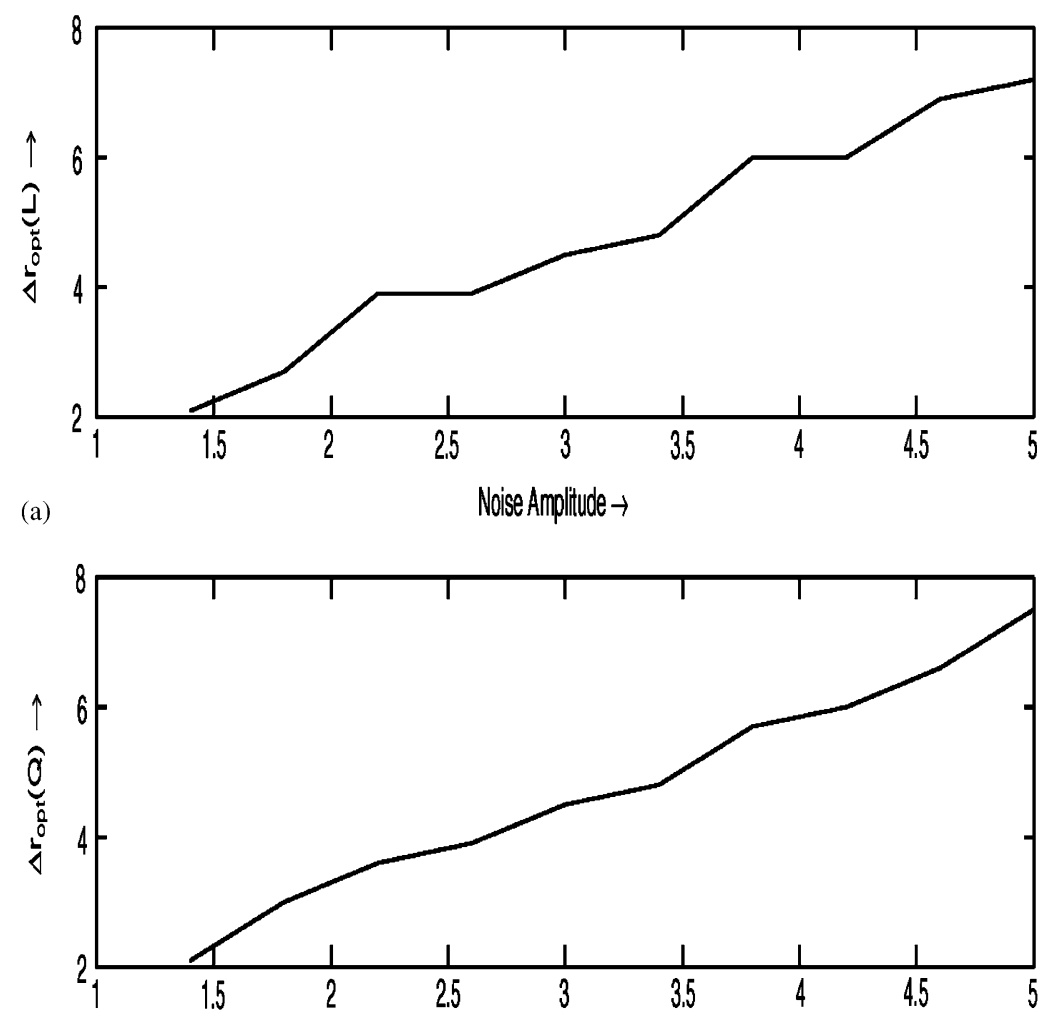

(b)

Noise Amplitude $\rightarrow$

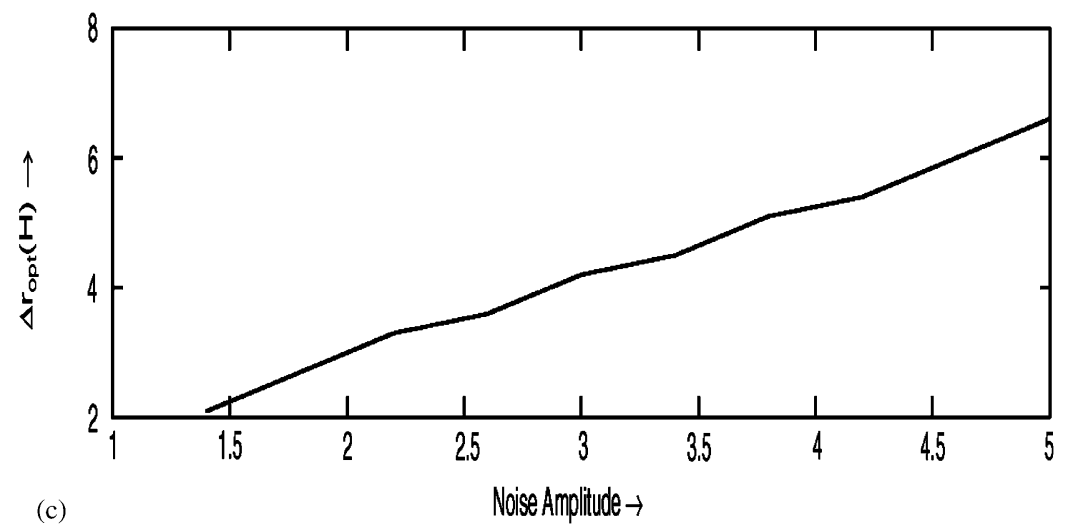

Fig. 5. The variation of the optimum bandwidth $\left(\Delta r_{\mathrm{opt}}\right)$ obtained from the indices of fuzziness (see Fig. 4(b)). (a) The variation of $\Delta r_{\text {opt }}$ (corresponding to the maxima of $L$ ) with the noise amplitude, (b) The variation of $\Delta r_{\text {opt }}$ (corresponding to the maxima of $Q$ ) with the noise amplitude, (c) The variation of $\Delta r_{\text {opt }}$ (corresponding to the maxima of $H$ ) with the noise amplitude. 
Alternatively,

$$
\Delta r_{\mathrm{opt}}=2 \frac{\left\langle\delta_{\mathbf{x}}^{2}\right\rangle_{\Omega}}{\left\langle\delta_{\mathbf{x}}\right\rangle_{\Omega}},
$$

where $\langle\cdot\rangle$ represents the sample average over the set of supporting points $\Omega$. For a large number of points, we can approximate $\Delta r$ (from the law of large number) as

$$
\Delta r_{\mathrm{opt}}=2 \frac{E_{\Omega}\left(\delta_{\mathbf{x}}^{2}\right)}{E_{\Omega}\left(\delta_{\mathbf{x}}\right)},
$$

where the expectation $E_{\Omega}(\cdot)$ is computed over the set of supporting points. Let $p\left(\delta_{\mathbf{x}}\right)$ represent the density function of $\delta_{\mathbf{x}}$. Let the point set in $\Omega$ be such that $\delta_{\mathbf{x}}$ follow a triangular distribution which can be ideally modeled by triangular membership function as in (37). Then

$$
\begin{aligned}
p\left(\delta_{\mathbf{x}}\right) & =k\left(1-\frac{\delta_{\mathbf{x}}}{\sigma}\right) & & \text { for } \delta_{\mathbf{x}}<\sigma, \\
& =0 & & \text { otherwise }
\end{aligned}
$$

where $k$ is the normalizing constant and $\sigma$ is the maximum deviation of a point from the mean of the distribution. Let us assume that $\Delta r_{\mathrm{opt}} \geqslant \sigma$. Then

$$
\begin{aligned}
\Delta r_{\mathrm{opt}} & =2 \frac{\int_{0}^{\sigma} \delta_{\mathbf{x}}^{2}\left(1-\delta_{\mathbf{x}} / \sigma\right) \mathrm{d} \delta_{\mathbf{x}}}{\int_{0}^{\sigma} \delta_{\mathbf{x}}\left(1-\delta_{\mathbf{x}} / \sigma\right) \mathrm{d} \delta_{\mathbf{x}}} \\
& =\sigma .
\end{aligned}
$$

Let $\Delta r_{\mathrm{opt}} \leqslant \sigma$. Then

$$
\begin{aligned}
\Delta r_{\mathrm{opt}} & =2 \frac{\int_{0}^{\Delta r_{\mathrm{opt}}} \delta_{\mathbf{x}}^{2}\left(1-\delta_{\mathbf{x}} / \sigma\right) \mathrm{d} \delta_{\mathbf{x}}}{\int_{0}^{\Delta r_{\mathrm{opt}}} \delta_{\mathbf{x}}\left(1-\delta_{\mathbf{x}} / \sigma\right) \mathrm{d} \delta_{\mathbf{x}}} \\
& =2 \Delta r_{\mathrm{opt}} \frac{\frac{1}{3}-\frac{\Delta r_{\mathrm{opt}}}{4 \sigma}}{\frac{1}{2}-\frac{\Delta r_{\mathrm{opt}}}{3 \sigma}}
\end{aligned}
$$

i.e.,

$$
\Delta r_{\mathrm{opt}}=\sigma .
$$

In either case, it is observed that the quadratic entropy gets maximized when the bandwidth becomes equal to the maximum possible deviation of the points from the true model shape. In other words, in the case of triangular distribution, if the membership function is chosen in such a way that it has the same form as the distribution function then $\Delta r_{\text {opt }}$ is exactly the same as the maximum deviation of a point from the model shape.

Case II: Let us now consider the case of exponential distribution such that the membership function has also an exponential form given as:

$$
\mu_{\mathcal{M}}^{\Theta, \Delta r}(x, y)=\exp \left(-\frac{\delta_{\mathbf{x}}^{2}}{\Delta r^{2}}\right),
$$


The natural ambiguity measure, in this case, is Shannon's entropy which is given as

$$
H(\Theta, \Delta r)=-\sum_{(x, y) \in \mathbf{X}} \mu_{\mathcal{M}}^{\Theta, \Delta r}(x, y) \ln \mu_{\mathcal{M}}^{\Theta, \Delta r}(x, y) .
$$

For $\Delta r=\Delta r_{\mathrm{opt}}$, we have

$$
\frac{\partial H}{\partial \Delta r} \mid\left(\Delta r=\Delta r_{\text {opt }}\right)=0
$$

From Eqs. (40)-(42),

$$
\Delta r_{\mathrm{opt}}^{2}=\frac{\sum_{\mathbf{x}} \delta_{\mathbf{x}}^{4} \exp \left(-\frac{\delta_{\mathbf{x}}^{2}}{\Delta r_{\mathrm{opt}}^{2}}\right)}{\sum_{\mathbf{x}} \delta_{\mathbf{x}}^{2} \exp \left(-\frac{\delta_{\mathbf{x}}^{2}}{\Delta r_{\mathrm{opt}}^{2}}\right)} .
$$

For a large number of points in $\mathbf{X}, \Delta r_{\text {opt }}$ can be approximated as

$$
\Delta r_{\mathrm{opt}}^{2}=\frac{\int_{0}^{\infty} \delta_{\mathbf{x}}^{4} \exp \left(-\frac{\delta_{\mathbf{x}}^{2}}{\Delta r_{\mathrm{opt}}^{2}}\right) p\left(\delta_{\mathbf{x}}\right) \mathrm{d} \delta_{\mathbf{x}}}{\int_{0}^{\infty} \delta_{\mathbf{x}}^{2} \exp \left(-\frac{\delta_{\mathbf{x}}^{2}}{\Delta r_{\mathrm{opt}}^{2}}\right) p\left(\delta_{\mathbf{x}}\right) \mathrm{d} \delta_{\mathbf{x}}}
$$

Let $p\left(\delta_{\mathbf{x}}\right)=1 / \sqrt{2 \pi} \sigma \exp \left(-\delta_{\mathbf{x}}^{2} / 2 \sigma^{2}\right)$, and let $\Delta r_{\mathrm{opt}}=K \sigma$ where $K$ is a constant of proportionality. Integrating both numerator and the denominator, it can be shown that

$$
\Delta r_{\mathrm{opt}}^{2}=\frac{3 K^{2}}{K^{2}+2} \sigma^{2}
$$

i.e.,

$$
K^{2}=\frac{3 K^{2}}{K^{2}+2}
$$

Solving for $K$,

$$
\Delta r_{\mathrm{opt}}=\sigma \text {. }
$$

In other words, the optimum bandwidth $(\Delta r)$, detected from the maxima of $H$, follow a linear relationship with the standard deviation of the distances of the points from the model shape.

\subsection{For gray-tone images}

The accumulator values, in the case of gray-tone images, depend not only on the bandwidth $\Delta r$ but also on the central gray value $l_{0}$ for a given $\Delta l$ (Eq. (23)). The optimum values of $\Delta r$ and $l_{0}$ for a given $\Delta l$ can be selected in the same way as in the case of a two-tone image. 
In the case of a graytone, first the optimum value of the parameter $l_{0}$ is determined from the maxima in the accumulator values just in the same way as the parameter values $\Theta$. The local maxima for different values of $l_{0}$ for a given $\Delta r$ is determined along with the parameter set $\Theta$. Next the ambiguity is measured for the optimum $l_{0}$ and the parameter set $\Theta$. It is found that $l_{0 \mathrm{opt}}$ is independent of the selection of the bandwidth $\Delta r$ so long as there are sufficient number of points in the supporting set. Mathematically, $l_{0 \mathrm{opt}}$ is such that

$$
\left.\frac{\partial A\left(\Theta, \Delta r, l_{0}\right)}{\partial l_{0}}\right|_{l_{0}=l_{0 \mathrm{opt}}}=0 .
$$

Considering a large number of points in the supporting set and a Gaussian form of the membership function

$$
\int\left(l-l_{0}\right) \exp \left(-\frac{\left(l-l_{0}\right)^{2}}{\Delta l^{2}}\right) \exp \left(-\frac{\delta_{\mathbf{x}}^{2}}{\Delta r^{2}}\right) p\left(\delta_{\mathbf{x}}, l\right) \mathrm{d} \delta_{x} \mathrm{~d} l=0,
$$

where $p\left(\delta_{\mathbf{x}}, l\right)$ is the spatial distribution of the pixels and their intensity values. If the spatial distribution and the intensity values are assumed to be independent Gaussian then

$$
p\left(\delta_{\mathbf{x}}, l\right)=\frac{1}{2 \pi \sigma_{\mathbf{x}} \sigma_{l}} \exp \left(-\frac{\delta_{\mathbf{x}}^{2}}{2 \sigma_{\mathbf{x}}^{2}}\right) \exp \left(-\frac{\left(l-\mu_{l}\right)^{2}}{2 \sigma_{l}^{2}}\right),
$$

where $\mu_{l}$ and $\sigma_{l}$ are the mean and standard deviation of the gray value distribution of the model candidate shape. Similarly, $\sigma_{\mathbf{x}}$ is the standard deviation of the pixels from the model candidate shape. After simplification, it can be found that

$$
l_{0}=\mu_{l}^{\prime},
$$

where

$$
\mu_{l}^{\prime}=\frac{\mu_{l}+\frac{2 l_{0} \sigma_{l}^{2}}{\Delta l^{2}}}{1+\frac{2 \sigma_{l}^{2}}{\Delta l^{2}}} .
$$

From Eqs. (51) and (52), it can be found that

$$
l_{0}=\mu_{l},
$$

i.e., the average gray value obtained from the local maxima of $A\left(\Theta, \Delta r, l_{0}\right)$ is independent of the choice of $\Delta l$ and $\Delta r$, and is equal to the mean of the pixel intensity distribution around the model shape.

The information content in a gray image can be quantified, as in the case of a two-tone image, by various measures namely, linear index of fuzziness, quadratic entropy, Shannon's entropy or various other measures given as

$$
\begin{aligned}
& L\left(\Theta, \Delta r, l_{0}, \Delta l\right)=\sum_{(x, y) \in \mathbf{I}} \min \left\{\mu_{\mathcal{M}}^{\Theta, \Delta r, l_{0}, \Delta l}(x, y), 1-\mu_{\mathcal{M}}^{\Theta, \Delta r, l_{0}, \Delta l}(x, y)\right\}, \\
& Q\left(\Theta, \Delta r, l_{0}, \Delta l\right)=\sum_{(x, y) \in \mathbf{I}} \mu_{\mathcal{M}}^{\Theta, \Delta r, l_{0}, \Delta l}(x, y)\left(1-\mu_{\mathcal{M}}^{\Theta, \Delta r, l_{0}, \Delta l}(x, y)\right)
\end{aligned}
$$


and

$$
H\left(\Theta, \Delta r, l_{0}, \Delta l\right)=-\sum_{(x, y) \in \mathbf{I}} \mu_{\mathcal{M}}^{\Theta, \Delta r, l_{0}, \Delta l}(x, y) \ln \mu_{\mathcal{M}}^{\Theta, \Delta r, l_{0}, \Delta l}(x, y) .
$$

The optimum value of $\Delta r$ for a given $l_{0}$ and $\Delta l$ can be obtained from the location of maxima of $L$ or $Q$ or $H$. However, $l_{0}$ and $\Delta l$ cannot be determined simultaneously. For a given $\Delta l, l_{0}$ can be determined from the maxima in $L$ or $Q$ or $H$. Thus in determining the optimum bandwidth and the average gray value, the maxima are found in $L, Q$ or $H$ for a specified $\Delta l$. Let us now provide a mathematical explanation of the optimum $\Delta r$ with the shape distortion.

Let us consider the case of exponential form of membership function such that

$$
\mu_{\mathcal{M}}^{\Theta, \Delta r, l_{0}, \Delta l}(x, y)=g(d(x, y), \Delta r) \cdot h_{1}\left(l(x, y), l_{0}, \Delta l\right)
$$

is given as

$$
\mu_{\mathcal{M}}^{\Theta, \Delta r, l_{0}, \Delta l}(x, y)=\exp \left(-\frac{\delta_{\mathbf{x}}^{2}}{\Delta r^{2}}\right) \exp \left(-\frac{\delta_{l}^{2}}{\Delta l^{2}}\right)
$$

where $\delta_{\mathbf{x}}$ is the deviation of a point $\mathbf{x}=(x, y)$ from the model shape $M$ and $\delta_{l}$ is the deviation of the gray value of the pixel from the average gray value of the shape. Note that, it has been assumed in the membership function that the gray level variation is independent of the distance of a point from the model shape. More rigorous membership function reflecting the dependency of the gray value on the distance of a point from the model shape may also be investigated. However, for sake of simplicity, the independence assumption is made.

As in the case of two-tone images, a natural ambiguity measure is Shannon's entropy given by Eq. (28). For the optimum bandwidth, i.e., $\Delta r=\Delta r_{\mathrm{opt}}$, we have

$$
\left.\frac{\partial H}{\partial \Delta r}\right|_{\Delta r=\Delta r_{\mathrm{opt}}}=0 \text {. }
$$

Simplifying we get

$$
\Delta r_{\mathrm{opt}}^{2}=\frac{\sum_{\mathbf{x}} \delta_{\mathbf{x}}^{4} \exp \left(-\frac{\delta_{\mathbf{x}}^{2}}{\Delta r_{\mathrm{opt}}^{2}}-\frac{\delta_{l}^{2}}{\Delta l^{2}}\right)}{\sum_{\mathbf{x}} \delta_{\mathbf{x}}^{2}\left(1-\frac{\delta_{l}^{2}}{\Delta l^{2}}\right) \exp \left(-\frac{\delta_{\mathbf{x}}^{2}}{\Delta r_{\mathrm{opt}}^{2}}-\frac{\delta_{l}^{2}}{\Delta l^{2}}\right)} .
$$

For a large number of points, $\Delta r_{\text {opt }}$ can be approximated as

$$
\Delta r_{\mathrm{opt}}^{2}=\frac{\int \delta_{\mathbf{x}}^{4} \exp \left(-\frac{\delta_{\mathbf{x}}^{2}}{\Delta r_{\mathrm{opt}}^{2}}-\frac{\delta_{l}^{2}}{\Delta l^{2}}\right) p\left(\delta_{\mathbf{x}}, \delta_{l}\right) \mathrm{d} \delta_{\mathbf{x}} \mathrm{d} \delta_{l}}{\int \delta_{\mathbf{x}}^{2}\left(1-\frac{\delta_{l}^{2}}{\Delta l^{2}}\right) \exp \left(-\frac{\delta_{\mathbf{x}}^{2}}{\Delta r_{\mathrm{opt}}^{2}}-\frac{\delta_{l}^{2}}{\Delta l^{2}}\right) p\left(\delta_{\mathbf{x}}, \delta_{l}\right) \mathrm{d} \delta_{\mathbf{x}} \mathrm{d} \delta_{l}} .
$$


Considering the pixel intensities are distributed such that $\delta_{\mathbf{x}}$ and $\delta_{l}$ are independent Gaussian, i.e.,

$$
p\left(\delta_{\mathbf{x}}, \delta_{l}\right)=\frac{1}{2 \pi \sigma_{\mathbf{x}} \sigma_{l}} \exp \left(-\frac{\delta_{\mathbf{x}}^{2}}{2 \sigma_{\mathbf{x}}^{2}}\right) \exp \left(-\frac{\delta_{l}^{2}}{2 \sigma_{l}^{2}}\right),
$$

where $\sigma_{\mathbf{x}}$ and $\sigma_{l}$ are the standard deviations of the shape distortion and gray level variation. Note that, as mentioned before, the gray level variation may not be independent of the distance of a pixel from the model shape. However, for white noise injected into the intensity profile of a distorted two-tone shape, $\delta_{l}$ will behave independently of $\delta_{\mathbf{x}}$. Integrating both numerator and denominator of Eq. (61),

$$
\Delta r_{\mathrm{opt}}^{2}=\frac{3\left(k_{l}^{2}+2\right)}{k_{l}^{2}+1} \cdot \frac{k_{\mathbf{x}}^{2}}{k_{\mathbf{x}}^{2}+2} \cdot \sigma_{\mathbf{x}}^{2},
$$

where $k_{\mathbf{x}}=\Delta r_{\mathrm{opt}} / \sigma_{\mathbf{x}}$ and $k_{l}=\Delta l / \sigma_{l}$. Considering $\Delta r_{\mathrm{opt}}=k_{\mathbf{x}} \sigma_{\mathbf{x}}$,

$$
k_{\mathbf{x}}^{2}=\frac{k_{l}^{2}+4}{k_{l}^{2}+1} .
$$

It is seen from (64) that $1 \leqslant k_{\mathbf{x}} \leqslant 2$, i.e., $\sigma_{\mathbf{x}} \leqslant \Delta r_{\text {opt }} \leqslant 2 \sigma_{\mathbf{x}} . \Delta r_{\text {opt }}$ is very close to $2 \sigma_{\mathbf{x}}$ when $k_{l}$ is very small, i.e., $\Delta l$ is very small. On the other hand, $\Delta r_{\text {opt }}$ approaches $\sigma_{\mathbf{x}}$ when $\Delta l$ is very large. In other words, if very large tolerance is allowed in the membership function then $\Delta r_{\mathrm{opt}}$ approaches the standard deviation of the shape distortion. On the other hand, a large tolerance in gray level variation will not be able to reflect the average gray value of the model shape.

Using the similar reasoning for the selection $\Delta l$, it can be found that

$$
k_{l}^{2}=\frac{k_{\mathbf{x}}^{2}+4}{k_{\mathbf{x}}+1} .
$$

Considering (64) and (65), it is seen that the optimum bandwidth is $\Delta r_{\mathrm{opt}}=\sqrt{2} \sigma_{\mathbf{x}}$ and the optimum tolerance in gray value is $\Delta l=\sqrt{2} \sigma_{l}$. However, it is difficult to optimize both $\Delta r$ and $\Delta l$ simultaneously from the maxima of the ambiguity measure. Therefore, for practical consideration, $\Delta l$ is specified by the user and the bandwidth is determined for a given tolerance in the gray value.

\section{Overall algorithm and experimental results}

As discussed in the previous section, the accumulator values need to be computed for a certain range of parameter values and a certain range of gray values (in the case of gray level image) for different values of $\Delta r$. It is very time consuming to maintain such a high dimensional array. Instead, for a sufficiently large value of $\Delta r$, the peaks in the accumulator space are first determined (in the case of gray level image, the accumulator also accommodates the gray value). After computing the local peaks, the $\Delta r$ is varied and the optimum $\Delta r$ is found from the ambiguity measures for each peak individually.

Let us now summarize the overall algorithm for obtaining the fuzzy Hough transform. The fuzzy Hough transform algorithm can be described in the same way as the classical Hough transform algorithm (Section 2) with additional steps for selecting the optimal $\Delta r$ and $l_{0}$. 


\subsection{For two-tone images}

Step 1: Quantize the accumulator space in the limits

$$
\theta_{1_{\min }} \leqslant \theta_{1} \leqslant \theta_{1_{\max }}, \theta_{2_{\min }} \leqslant \theta_{2} \leqslant \theta_{2_{\max }}, \ldots, \theta_{n_{\min }} \leqslant \theta_{n} \leqslant \theta_{n_{\max }} .
$$

Fix a threshold $A_{0}$ in the accumulator space.

Set $\Delta r=\Delta r_{0}$, where $\Delta r_{0}$ is a reasonably high value close to $\Delta r_{\max }$ causing a high amount of smoothing. Initialize the accumulator $A(\Theta)$ to zero.

Step 2: For each object pixel $(x, y)$ in the image compute $\mu_{\mathcal{M}}^{\Theta, \Delta r}(x, y)$.

Compute

$$
A(\Theta)=A(\Theta)+\mu_{\mathcal{M}}^{\Theta, \Delta r}(x, y) .
$$

Step 3: Determine the peaks in $A(\Theta)$ such that $A(\Theta)>$ threshold where threshold is a minimum threshold in the accumulator space.

Step 4: Quantize the bandwidth $\Delta r$ in the limits

$$
\Delta r_{\min } \leqslant \Delta r \leqslant \Delta r_{\max }
$$

Define an array $V(\Theta, \Delta r)$.

Set the starting value of $\Delta r$ to its minimum, i.e., $\Delta r=\Delta r_{\min }$.

Step 5: Initialize $V(\Theta, \Delta r)$ to zero.

For each $\Theta$ if $A(\Theta)$ is a peak, compute

$$
V(\Theta, \Delta r)=V(\Theta, \Delta r)+a(\Theta, \Delta r),
$$

where $a(\Theta, \Delta r)$ is an ambiguity measure given by linear index of fuzziness or quadratic entropy or Shannon's entropy.

Step 6: Get the next value of quantized $\Delta r$.

Repeat the steps 5 and 6 until $\Delta r$ becomes equal to $\Delta r_{\max }$.

Step 7: Find the set of local maxima in $V(\Theta, \Delta r)$. These local maxima indicate the set of optimum bandwidths $\Delta r$ quantifying the distortion present in the shapes.

\subsection{For gray-tone images}

The algorithm for computing the FHT for gray-tone images is similar to that for two-tone images except that the additional parameters $l_{0}$ and $\Delta l$ need to be incorporated in order to capture the average gray value and the gray level variation. The algorithm is as follows.

Step 1: Quantize the accumulator space in the limits

$$
\theta_{1_{\min }} \leqslant \theta_{1} \leqslant \theta_{1_{\max }}, \theta_{2_{\min }} \leqslant \theta_{2} \leqslant \theta_{2_{\max }}, \ldots, \theta_{n_{\min }} \leqslant \theta_{n} \leqslant \theta_{n_{\max }} .
$$

Quantize the gray value $l_{0}$ in the range

$$
l_{0 \min } \leqslant l_{0} \leqslant l_{0 \max } .
$$

Fix a threshold $A_{0}$ in the accumulator space.

Fix the parameter $\Delta l$. 
Fix $\Delta r$ close to $\Delta r_{\max }$.

Initialize the accumulator space $A\left(\Theta, l_{0}\right)$ to zero.

Step 2: For each pixel $(x, y)$ in the image compute $\mu_{\mathcal{M}}^{\Theta, \Delta r, l_{0}}(x, y)$.

Compute

$$
A\left(\Theta, l_{0}\right)=A\left(\Theta, l_{0}\right)+\mu_{\mathcal{M}}^{\Theta, \Delta r, l_{0}}(x, y) .
$$

Step 3: Determine the peaks in $A\left(\Theta, l_{0}\right)$ such that for each peak $A\left(\Theta, l_{0}\right)>A_{0}$.

Step 4: Quantize the bandwidth $\Delta r$ in the limits

$$
\Delta r_{\min } \leqslant \Delta r \leqslant \Delta r_{\max } .
$$

Define an array $V\left(\Theta, \Delta r, l_{0}\right)$.

Set the starting value of $\Delta r$ to its minimum, i.e., $\Delta r=\Delta r_{\min }$.

Step 5: Set $V\left(\Theta, \Delta r, l_{0}\right)$ to zero.

For each $\Theta$ and $l_{0}$ if $A\left(\Theta, l_{0}\right)$ is a peak then compute

$$
V\left(\Theta, \Delta r, l_{0}\right)=V\left(\Theta, \Delta r, l_{0}\right)+a\left(\Theta, \Delta r, l_{0}\right)
$$

where $a(\cdot)$ is the linear index of fuzziness or quadratic entropy or Shannon's entropy.

Step 6: Get the next quantized value $\Delta r$.

Repeat the steps 5 and 6 until $\Delta r$ becomes equal to $\Delta r_{\max }$.

Step 7: Find the set of local maxima in $V\left(\Theta, \Delta r, l_{0}\right)$. These local maxima indicate the bandwidths $\Delta r$ quantifying the distortion present in the shapes.

\subsection{Experimental results}

The effectiveness of the proposed fuzzy Hough transform (FHT) algorithm is illustrated on different synthetically generated binary and gray images as shown in Figs. 6-8. In Fig. 6, points are generated from more than one straight line segment. The points are randomly shifted horizontally by a noise amplitude varying from 1.0 to 5.0. The parameter $\Delta r$ is varied from 0.9 to 6.0 in steps of 0.3 . The parameters $\alpha$ and $r$ (Eq. (3)) are quantized in steps of unity.

The threshold in the accumulator space is chosen as 40 . The different straight lines are identified along with their bandwidths as shown in Fig. 6. The core lines denote the identified straight line segments. The bandwidths, quantifying the distortion, are specified by the region between the parallel line segments around the core line.

Fig. 7 illustrates the performance of the algorithm in identifying two distorted circles. Points are generated from two different circles with radii 17 and 23, respectively. The points are randomly shifted horizontally and vertically with noise amplitudes 2 and 5, respectively. $\Delta r$ is varied from 1.5 to 6.0. The parameter values ( $a, b$ and $r$ in Eq. (4)) are quantized in steps of unity. The different circles, identified by the proposed FHT algorithm, are shown in Fig. 8(a) and (b) respectively. The core lines indicate the identified circles. The vagueness in the shape description, as quantified by the bandwidth, are indicated by the region within two concentric circles around the core lines.

Let us now demonstrate the performance of the FHT algorithm on a $50 \times 50$ gray image as shown in Fig. 9. Two line segments are generated with maximum gray levels 25 and 250 , respectively. The pixels on the lines are randomly shifted (both horizontally and vertically) by 2 pixels. The points on the faint 

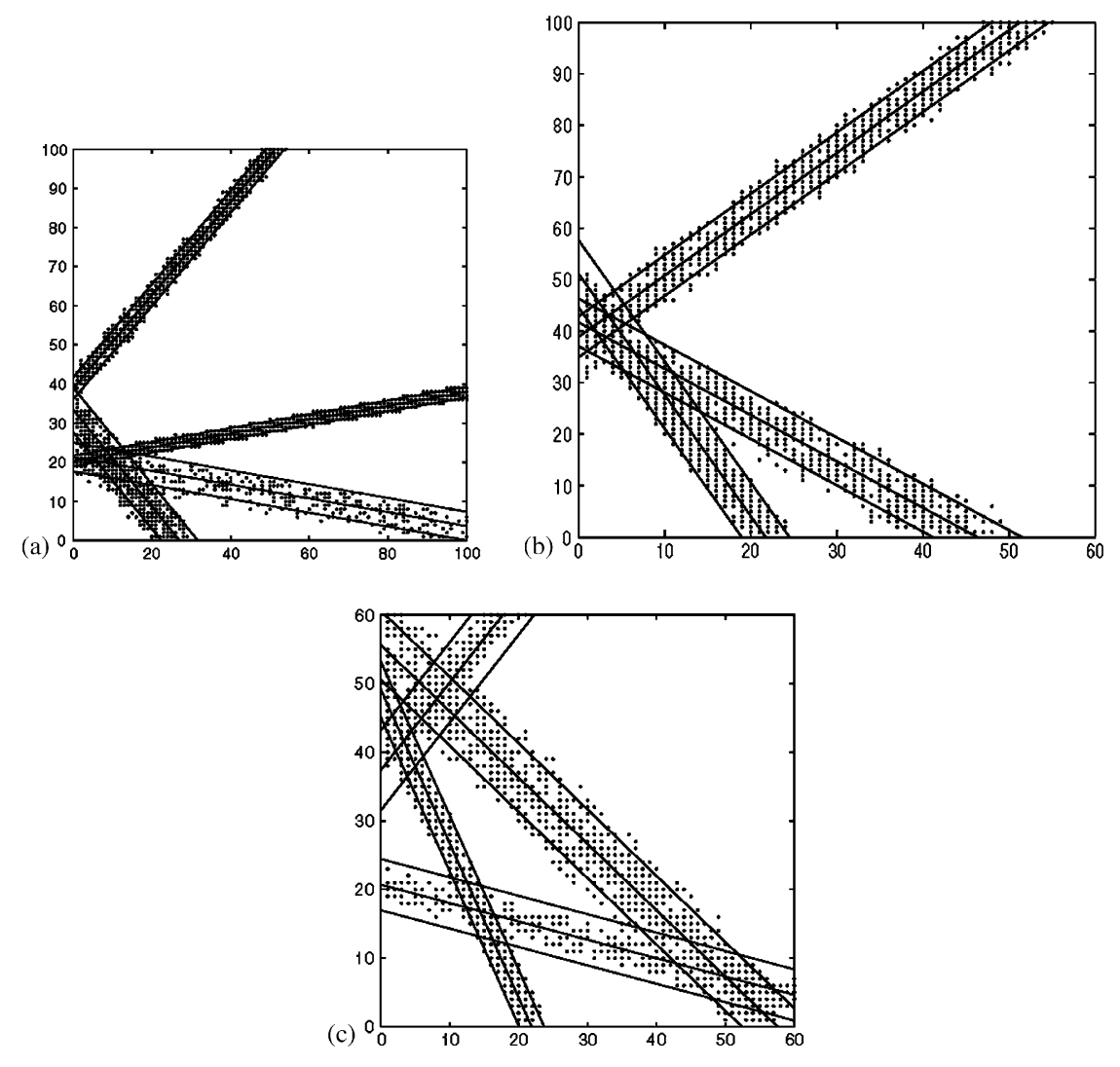

Fig. 6. The lines detected by the fuzzy Hough transform algorithm along with the identified bandwidths specifying the amount of distortions in the shapes. For each line segment (bar), the central line (core line) indicates the detected line segment and the identified bandwidth is specified by the region enclosed by two segments parallel to the core line: (a) points are generated from four different straight lines with noise amplitude equal to 1.0, 2.0, 3.0 and 4.0 respectively; (b) points are generated from three different straight line segments with noise amplitude 2.0, 3.0 and 4.0 respectively; (c) points are generated from four different straight line segments with noise amplitude 2.0, 3.0 and 5.0, respectively.

line segment are then convolved with Gaussian window of standard deviation ( $\sigma$ ) equal to 3.0. Finally the gray image is contaminated with randomly generated (uniformly distributed) noisy pixels with gray values ranging from 0 to 30. It can be seen from Fig. 8(a) that the maximum gray value of the faint line is less than the maximum gray value of the noisy pixels.

In computing the FHT, $\Delta r$ is varied from 1.5 to 6.0 in steps of 0.3 . The parameters $\alpha$ and $r$ (Eq. (3)) are quantized in steps of unity. The gray value $l_{0}$ (Eq. (23)) is quantized in steps of 3 . The parameter $\Delta l$ is chosen as 10 . The threshold in the accumulator space is selected as 50 . The line segments (bars) identified in the gray image are shown in Fig. 8(b). The lines are detected along with their widths $\Delta r$ and average gray levels. It may be noted here that the conventional segmentation algorithms (e.g., gray level thresholding) will fail in separating the faint line from the noisy pixels since the gray level distribution of the faint line is embedded in that of the noisy pixels. 

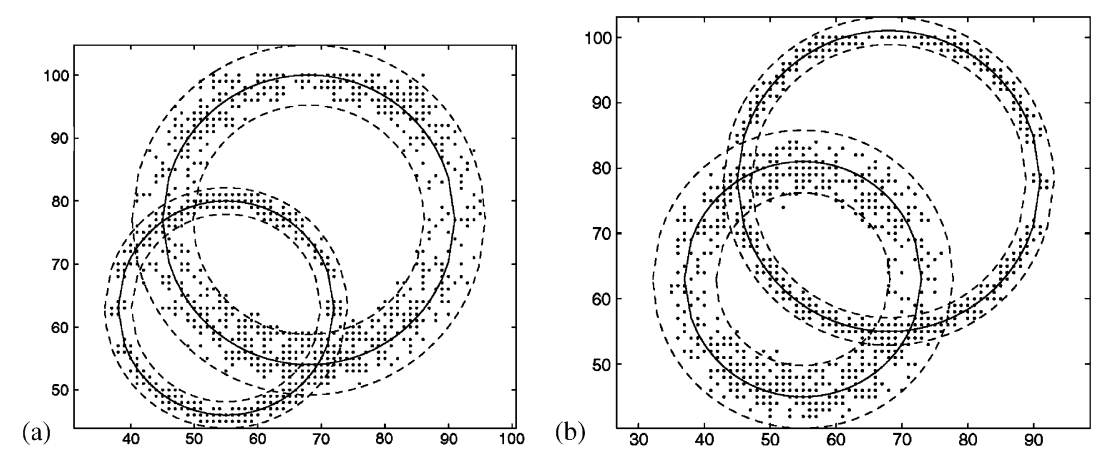

Fig. 7. The circles detected by the fuzzy Hough transform algorithm along with the bandwidths specifying the amount distortions in the shapes. For each circle, the central line (core line) indicates the true circle detected and the bandwidth is specified by the region enclosed within two circles concentric to the core one: (a) the bigger circle is distorted by a noise amplitude of 5.0 and the smaller one by 2.0; (b) the bigger circle is distorted by a noise amplitude 2.0 and the smaller one by 5.0.
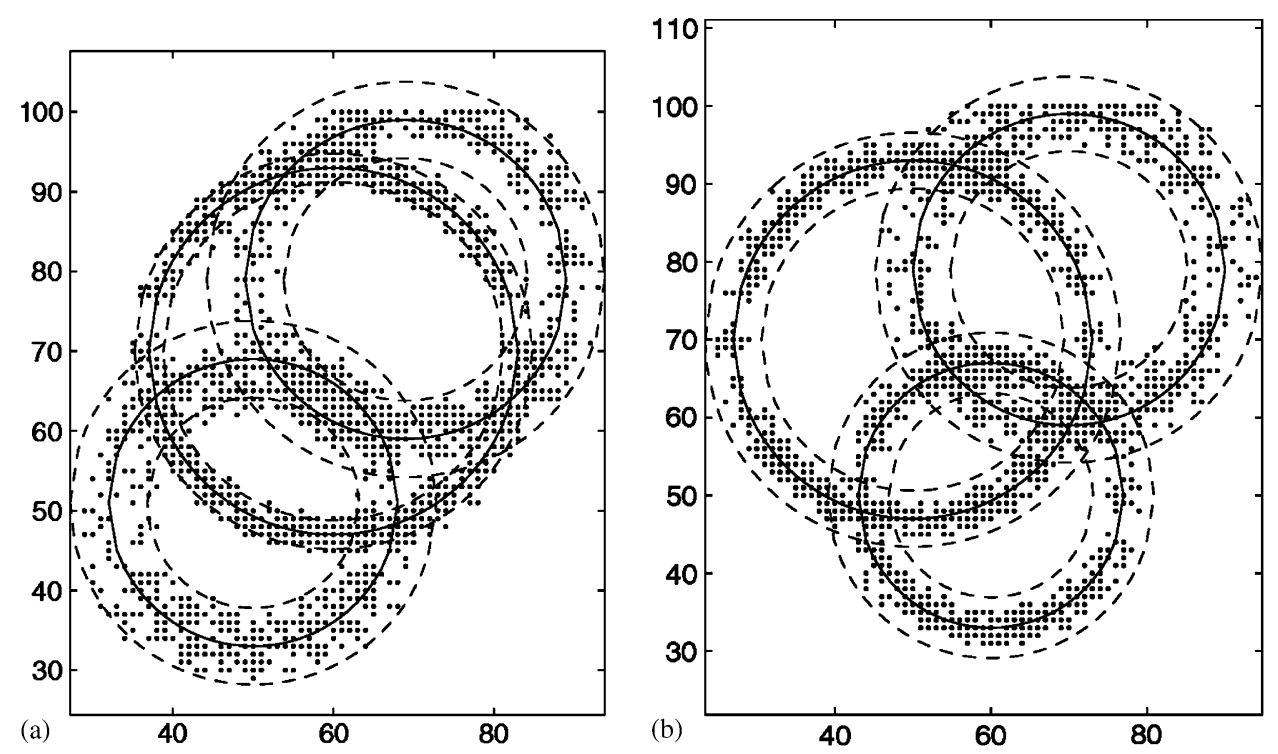

Fig. 8. The circles detected by the fuzzy Hough transform algorithm along with the bandwidths specifying the amount distortions in the shapes. For each circle, the central line (core line) indicates the true circle detected and the bandwidth is specified by the region enclosed within two circles concentric to the core one: (a) the circles are distorted by noise amplitudes 2.0 and 5.0 ; (b) the circles are distorted by noise amplitudes 3.0, 4.0 and 5.0, respectively.

\section{Conclusions}

A generalized framework of the fuzzy Hough transform is described in this paper. First, a set theoretic interpretation of the Hough transform is provided and then it is extended to a fuzzy set theoretic framework in order to take care of the impreciseness or ill-definedness in shapes. Although, the way of computing the FHT is analogous to that of the earlier methods [12,22], the scheme described for quantifying the 

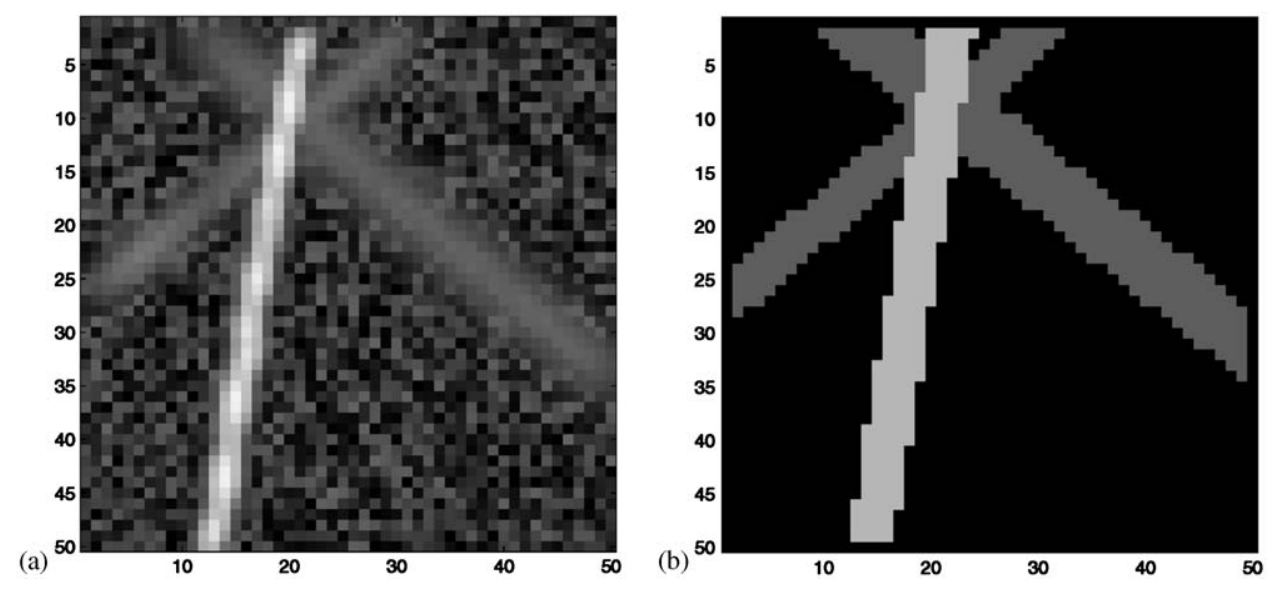

Fig. 9. (a) The original noisy gray image. (b) The lines identified by the FHT algorithm from gray images along with the bandwidth (specifying the distortion) and average gray value of the shapes.

amount of distortion present in the shapes is novel. The FHT algorithm is also able to handle gray level images unlike the previous methods.

In the case of gray level images, the algorithm not only finds out the distortion of the shapes but also specifies the average gray level of the shapes. The algorithm is found to be robust in the presence of noise, and is able to extract shapes even where the gray level distribution of the shapes is embedded in that of the noisy pixels. Note that, the existing global thresholding algorithms will fail in such situations. The method of generalization of the FHT algorithm to gray level gives rise to a segmentation scheme which can further be extended to take care of arbitrary shapes in the future. The proposed fuzzy Hough transform model can also be extended to a neurofuzzy framework to extract the parametric shapes in an unsupervised mode by the guidelines proposed in $[6,3,4]$.

In this paper, we provide mostly a theoretical analysis of the fuzzy Hough transform, and a guideline for automatic selection of the bandwidth of both shape parameter quantification and gray value classification. Note that, the gray value classification performed here is different from the gray level thresholding in the sense that the gray value classification occurs simultaneously with the shape extraction. In other words, the mean gray value of a structure depends on the localization of the structure itself. The localization of the structure and the gray value classification are inter-dependant as shown in the theoretical analysis. This can be further investigated for real-life images in the future. A possible localization in the real-life images can also be performed with the help of inverse Hough transform as provided in [15].

\section{References}

[1] D.H. Ballard, Generalizing the Hough transform to detect arbitrary shapes, Pattern Recognition 13 (1981) 111-122.

[2] D. Ballard, C. Brown, Computer Vision, Prentice-Hall Inc., Englewood Cliffs, NJ, 1982.

[3] J. Basak, Learning Hough transform: a neural network model, Neural Comput. 13 (2001) 651-676.

[4] J. Basak, A. Das, Hough transform network: a neural network for learning parametric structures, Neurocomputing 51 (2003) 125-145.

[5] J. Basak, S.K. Pal, PsyCOP: a psychologically motivated connectionist system for object perception, IEEE Trans. Neural Networks 6 (1995) 1337-1354. 
[6] J. Basak, S.K. Pal, Hough transform network, Electron. Lett. 35 (1999) 577-578.

[7] J. Basak, N.R. Pal, S.K. Pal, A connectionist system for learning and recognition of structures, Neural Networks 8 (1995) 643-657.

[8] J.R. Bergen, H. Shvaytser, A probabilistic algorithm for computing Hough transforms, J. Algorithms 12 (1991) $639-656$.

[9] S. Bhandarkar, A fuzzy probabilistic model of the generalized Hough transform, IEEE Trans. System Man Cybernet. 24 (1994) 745-759.

[10] R.O. Duda, P.E. Hart, Use of Hough transform to detect lines and curves in pictures, Commun. ACM 15 (1972) 11-15.

[11] D. Geci, Fuzzy Hough transform, Ph.D. Thesis, M.S. Thesis, University of West Florida, Pensacola, FL, 1996.

[12] J.H. Han, L.T. Koczy, T. Poston, Fuzzy Hough transform, Pattern Recognition Lett. 15 (1994) 649-658.

[13] P.V.C. Hough, Method and means for recognizing complex patterns, US Patent 3069654, 1962.

[14] J. Illingworth, J. Kittler, A survey of the Hough transform, Comput. Vision, Graph. Image Process. 44 (1988) $87-116$.

[15] A.L. Kesidis, N. Papamarkos, On the gray-scale inverse Hough transform, Image Vision Comput. 18 (2000) $607-618$.

[16] N. Kiryati, Y. Eldar, A.M. Bruckstein, A probabilistic Hough transform, Pattern Recognition 24 (1991) 303-316.

[17] V.F. Leavers, The dynamic generalized Hough transform: its relationship to the probabilistic Hough transforms and an application to the concurrent detection of circles and ellipses, CVGIP: Image Understand. 56 (1992) 381-398.

[18] V.F. Leavers, Which Hough transform?, CVGIP: Image Understand. 58 (1993) 250-264.

[19] H. Li, M. Lavin, R.J.L. Master, Fast Hough transform: a hierarchical approach, Comput. Vision, Graphics, Image Process. 36 (1986) 139-161.

[20] R. Lo, W. Tsai, Gray-scale Hough transform for thick line detection in gray-scale images, Pattern Recognition 28 (1996) 647-661.

[21] P.L. Palmer, M. Petrou, J. Kittler, A Hough transform algorithm with a 2D hypothesis testing kernel, CVGIP: Image Understand. 58 (1993) 221-234.

[22] K.P. Philip, E.L. Dove, D.D. McPherson, N.L. Gotteiner, W. Stanford, K.B. Chandran, The fuzzy Hough transform-feature extraction in medical images, IEEE Trans. Med. Imaging 13 (1994) 235-240.

[23] A. Pietrowcew, Face detection in colour images using fuzzy Hough transform, Opto-Electron. Rev. 11 (2003) $247-251$.

[24] V. Shapiro, On the Hough transform of multi-level pictures, Pattern Recognition 29 (1996) 589-602.

[25] R. Soodamani, Z.Q. Liu, A novel fuzzy Hough transform for shape representation, in: Fuzzy Systems Proceedings 1998, IEEE World Congress on Computational Intelligence, vol. 2, 1998, pp. 1605-1608.

[26] R. Soodamani, Z.Q. Liu, A fuzzy Hough transform approach to shape description, Int. J. Image Graph. 2 (2002) $603-616$.

[27] T.Y. Tian, M. Shah, Recovering 3D motion of multiple objects using adaptive Hough transform, IEEE Trans. Pattern Anal. Mach. Intell. 19 (1997).

[28] L. Xu, E. Oja, Randomized Hough transform (rht): basic mechanisms, algorithms, and computational complexities, CVGIP: Image Understand. 57 (1993) 131-154. 